\title{
Scattering Coefficients of Mice Organs Categorized Pathologically by Spectral Domain Optical Coherence Tomography
}

\author{
Q. Q. Zhang, ${ }^{1}$ X. J. Wu, ${ }^{2}$ C. Wang, ${ }^{1}$ S. W. Zhu, ${ }^{1}$ Y. L. Wang, ${ }^{2}$ Bruce Z. Gao, ${ }^{3}$ and X.-C. Yuan ${ }^{4}$ \\ ${ }^{1}$ Institute of Modern Optics, Key Laboratory of Optical Information Science \& Technology, Ministry of Education of China, \\ Nankai University, Tianjin 300071, China \\ ${ }^{2}$ Nankai University Affiliated Hospital, Tianjin 300121, China \\ ${ }^{3}$ Department of Bioengineering and COMSET, Clemson University, Clemson, SC 29634, USA \\ ${ }^{4}$ Institute of Micro \& Nano Optics, Shenzhen University, Shenzhen 518060, China
}

Correspondence should be addressed to Y. L. Wang; yinlongwang@hotmail.com and X.-C. Yuan; xcyuan@nankai.edu.cn

Received 22 January 2014; Revised 19 March 2014; Accepted 19 March 2014; Published 13 April 2014

Academic Editor: Shiwu Zhang

Copyright (c) 2014 Q. Q. Zhang et al. This is an open access article distributed under the Creative Commons Attribution License, which permits unrestricted use, distribution, and reproduction in any medium, provided the original work is properly cited.

Differences in tissue density cause a variety of scattering coefficients. To quantify optical coherence tomography (OCT) images for diagnosis, the tissue's scattering coefficient is estimated by curve fitting the OCT signals to a confocal single backscattering mode. The results from a group of 30 mice show that the scattering coefficients of bone, skin, liver, brain, testis, and spleen can be categorized into three groups: a scattering coefficient between 1.947 and $2.134 \mathrm{~mm}^{-1}$ : bone and skin; a scattering coefficient between 1.303 and $1.461 \mathrm{~mm}^{-1}$ : liver and brain; a scattering coefficient between 0.523 and $0.634 \mathrm{~mm}^{-1}$ : testis and spleen. The results indicate that the scattering coefficient is tissue specific and could be used in tissue diagnosis.

\section{Introduction}

In spectral domain optical coherence tomography (SDOCT), a spectrometer is used to obtain high-resolution structural imaging through low coherence interferometry. The technique, which measures backscattered light generated by tissue, has great potential in disease diagnosis, including differentiation between benign and malignant lesions [1]. OCT typically employs near-infrared light to acquire micronscale cross-sectional images of biological samples [2]. Both normal and premalignant tissues in various organs have been imaged by OCTs. For example, ovarian cysts, masses, and abnormal tissues have been successfully imaged using a laparoscopic OCT [3]. Laryngeal dysplasia and malignancy have been imaged with OCT probes during laryngoscopy [4], and malignant and inflammatory lymph nodes have been differentiated by OCT [5]. To gain more diagnostic information such as distinction between normal and abnormal tissues (as in histological analysis), OCT images must be further analyzed [6-10]. In addition to providing highresolution morphological images, OCT can quantitatively provide the scattering coefficient $\left(\mu_{s}\right) . \mu_{s}$ is approximately the total attenuation coefficient $\left(\mu_{t}\right)$, which is the summation of $\mu_{s}$ and the negligible absorption coefficient $\left(\mu_{a}\right)$ and can be estimated by fitting the OCT A-scan data to a theoretical model [11-13]. Using a single parameter such as $\mu_{t}$ to characterize in vivo tissue (instead of reconstructing and subsequently analyzing an OCT image), we may take the advantage of clinical OCT's high speed in vivo scanning $[14,15]$ and high spatial resolution detection [16] to achieve real-time clinical diagnoses. In the study, we use quantitative spectral domain optical coherence tomography to explore the scattering coefficients of various tissues or organs. Our approach is to take the OCT data as a function of depth with $\mu_{t}$ as a characteristic parameter and then fit the OCT data obtained from an actual tissue to the function to determine $\mu_{s}[6-10]$.

\section{Principle and Methods}

2.1. SDOCT System. The SDOCT system used in this study is described in our previous publication [17], and a schematic of 


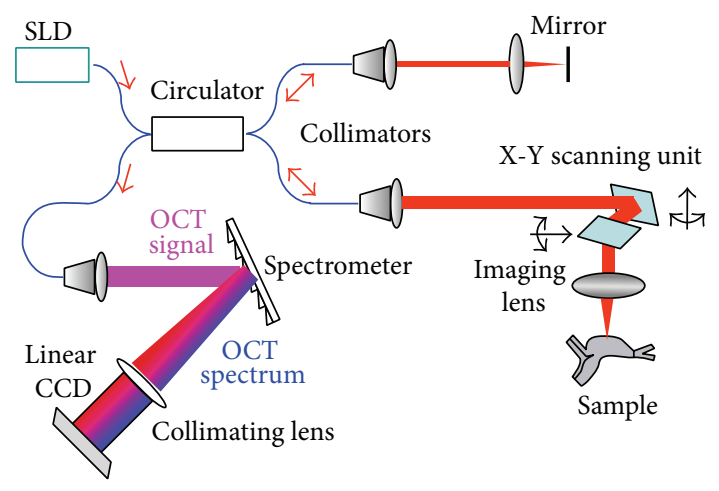

FIGURE 1: Schematic drawing of the SDOCT system.

the setup is shown in Figure 1. Light from a superluminescent diode (SLD) (S-840-B-I-20) with a bandwidth of $50 \mathrm{~nm}$ is split by a $50: 50$ fiber coupler into reference and sample arms. In the reference arm, the collimated beam from the singlemode fiber is reflected back from the reference mirror. In the sample arm, the collimated beam from the single-mode fiber is $x$ - $y$ scanned before being focused into the sample by a focusing lens ( $f=35 \mathrm{~mm}$ ). The OCT signal formed through interference of the reference and sample beams is detected by a custom spectrometer that consists of a fiber collimator, a diffraction grating (1200 lines/mm), an achromatic lens, and a line-scanning CCD (e2v AViiVA SM2 CL, 4096×1, $10 \mu \mathrm{m})$. The resultant OCT spectrum is recorded by a linear CCD camera and transferred to the computer to implement fast Fourier transform by the CCD's camera link. In our system, the axial resolution, determined by the bandwidth of the light source, is approximately $6.2 \mu \mathrm{m}$, and the lateral resolution, determined by the imaging system, is approximately $9.5 \mu \mathrm{m}$.

The SDOCT imaging size is 2048 axial $\times 600$ transverse pixels. To avoid a mirror image, light was focused on the surface of the scattering medium during imaging.

2.2. OCT Model. Two models are used for an OCT signal [1113, 18-22]: the single-backscattering model and the multiplescattering model. In the former, the light that has been backscattered only once contributes to the OCT signal, and the sample light is given by Beer's law:

$$
I(z) \propto \exp \left(-2 \mu_{t} z\right),
$$

where $I(z)$ is the intensity of the interference signal; the factor 2 is due to the round-trip attenuation. The conventional single-backscattering model, which is suitable for dynamic focusing, ignores the coherence gate-generated confocal effect. To account for this confocal effect to establish a more accurate model, an axial confocal PSF for the OCT system was introduced by Faber et al. [12]. The OCT signal containing the confocal PSF is given as follows:

$$
I(z) \propto \frac{\exp \left(-2 \mu_{t} z\right)}{\left(\left(z-z_{c f}\right) / z_{R}\right)^{2}+1},
$$

where $z_{c f}$ is the position of the focal plane and $z_{R}$ is the "apparent" Rayleigh length.

In our system, the numerical aperture of the imaging lens is 0.08 , which is low for a fixed-focus OCT system [10],
TABLE 1: Distribution of scattering coefficients.

\begin{tabular}{lcc}
\hline Organs & $\begin{array}{c}\text { Range of scattering } \\
\text { coefficient } \mu_{s}\left(\mathrm{~mm}^{-1}\right)\end{array}$ & $\begin{array}{c}\text { Mean scattering } \\
\text { coefficient } \mu_{s}\left(\mathrm{~mm}^{-1}\right)\end{array}$ \\
\hline Testis & $0.206-1.130$ & $0.523 \pm 0.036$ \\
Spleen & $0.201-1.141$ & $0.634 \pm 0.038$ \\
Brain & $0.729-1.797$ & $1.303 \pm 0.050$ \\
Liver & $0.870-1.994$ & $1.462 \pm 0.064$ \\
Bone & $1.130-2.763$ & $1.947 \pm 0.088$ \\
Skin & $1.517-2.742$ & $2.134 \pm 0.079$ \\
\hline
\end{tabular}

but provides sufficient focal zone for superficial scanning depth. The tissues we study are weakly scattering media $\left(\mu_{s}<\right.$ $8 \mathrm{~mm}^{-1}$ ), so the single-backscattering model can be used in the OCT signal analysis to estimate the scattering coefficient $[12,23]$. In our research, $\mu_{t}$ in (2) was replaced by $\mu_{s}$.

\section{Quantitative Analysis of Mouse Organs}

The study protocol for animal experimentation was approved by the Animal Protection Committee. Thirty identical $\mathrm{BALB} / \mathrm{c}$ mice were used to obtain bone, skin, liver, brain, testis, and spleen. These organs from each mouse were washed by immersion in $0.9 \%$ saline solution and placed on the objective stage for imaging. Based on the acquired SDOCT signals, we used the OCT model to study scattering coefficients in different organs. Depending on the size of each sample, we performed 10-20 measurements at evenly distributed locations. Each measurement included $600 \mathrm{~A}$ scans (each A-scan consisted of 4096 points) in which 3 evenly distributed regions of interest (ROIs), each consisting of 100 A-scans, were selected, as shown in Figure 2. A-scans in each ROI were averaged to estimate a scattering coefficient value. For each organ, we obtained a total of approximately 3 (ROIs) $\times 15$ (measurements/sample) $\times 30$ (samples $)=$ 1350 scattering coefficient values for statistical analysis. To avoid surface effects, the signals from $20 \mu \mathrm{m}$ below the tissue surface were used for curving fitting.

Figure 2 shows OCT images $(600 \times 200$ pixels $)$ and the corresponding curve fittings; a typical image is selected for each organ. The red boxes highlight the $100 \mathrm{~A}$-scans used to obtain an averaged A-scan for curve fitting. The differences in organ internal microstructures qualitatively reflected by the OCT images were quantified by the estimated $\mu_{s}$ values from Figures 2(a) to 2(f): $0.520 \mathrm{~mm}^{-1}, 0.639 \mathrm{~mm}^{-1}, 1.310 \mathrm{~mm}^{-1}$, $1.469 \mathrm{~mm}^{-1}, 1.944 \mathrm{~mm}^{-1}$, and $2.130 \mathrm{~mm}^{-1}$, respectively.

Figure 3 shows the histograms of $\mu_{s}$ values for each organ from the total measurements after discarding the maximum and minimum. Figure 3 shows that the distribution of $\mu_{s}$ conforms to a normal distribution, which is statistically meaningful. Because complete hair removal was difficult, the total number of OCT images for the skin is less than that for the other organs. However, the distribution of $\mu_{s}$ for the skin still conforms to a normal distribution.

The distribution of scattering coefficient $\mu_{s}$ is summarized in Table 1. Testis and spleen have smaller scattering coefficients. Brain and liver have larger scattering coefficients. Bone and skin have the largest scattering coefficients. 


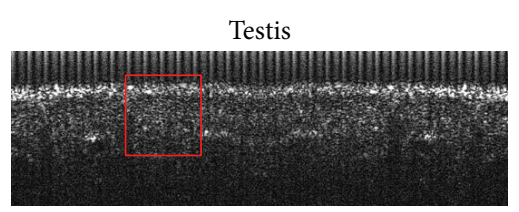

(a)

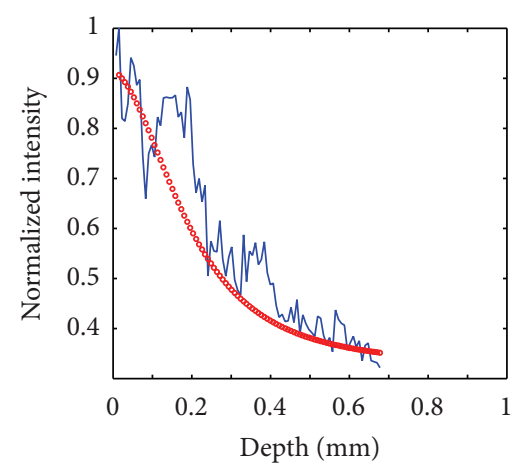

$\left(\mathrm{a}^{\prime}\right)$

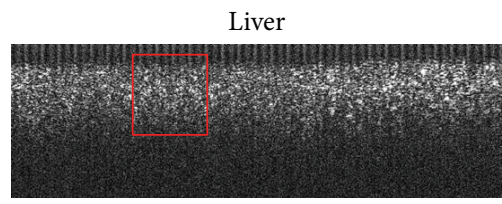

(d)

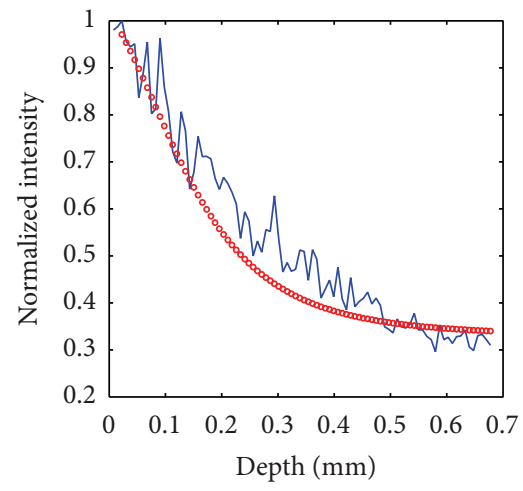

$\left(d^{\prime}\right)$

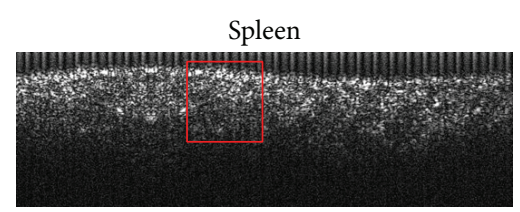

(b)

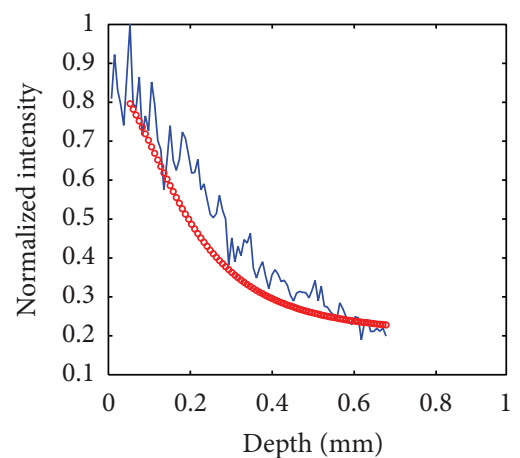

$\left(b^{\prime}\right)$

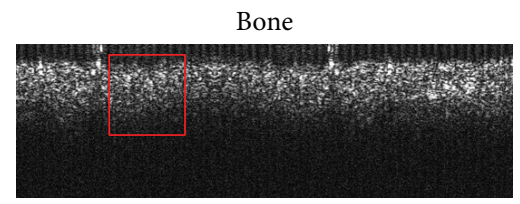

(e)

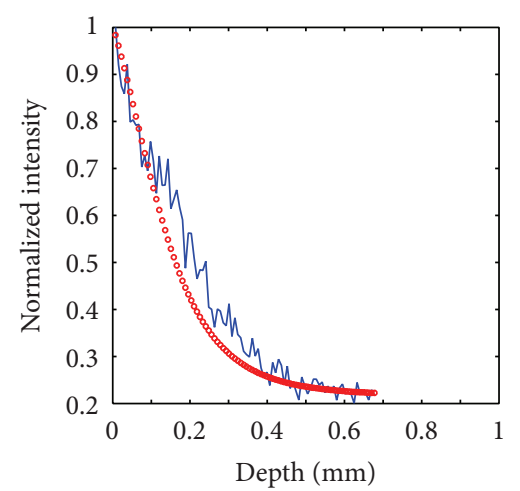

$\left(\mathrm{e}^{\prime}\right)$

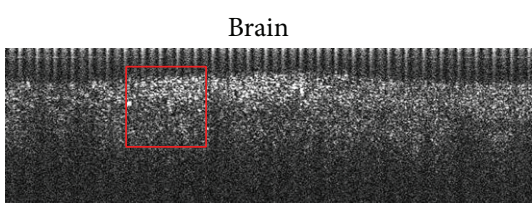

(c)

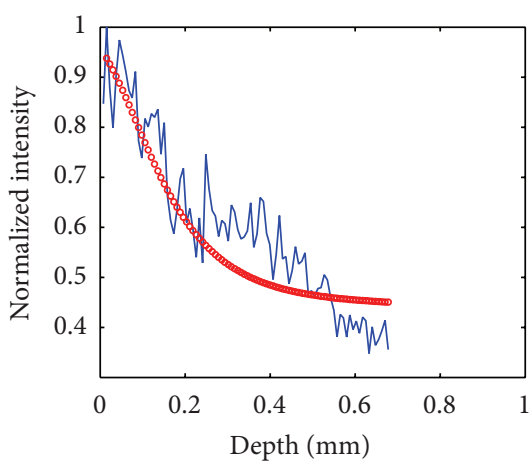

$\left(c^{\prime}\right)$

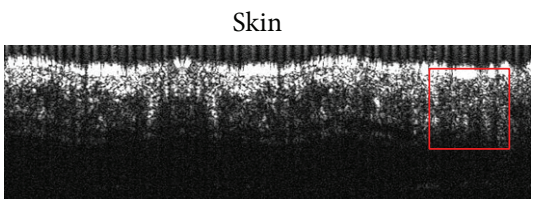

(f)

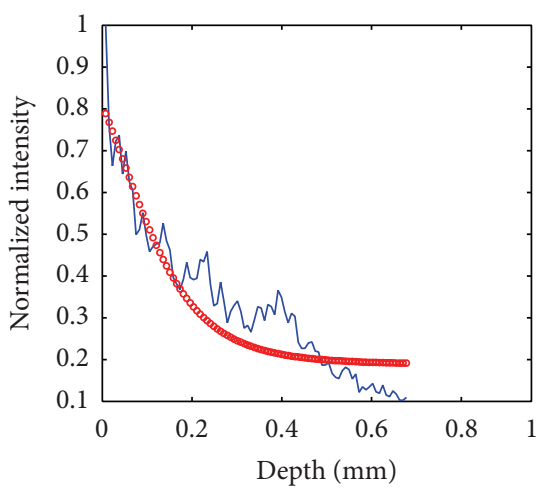

$\left(f^{\prime}\right)$

FIGURE 2: OCT images (a)-(f) and the corresponding curve fitting $\left(a^{\prime}\right)-\left(f^{\prime}\right)$.

The data shown in Table 1 are presented as a bar graph in Figure 4. It shows that three groups in terms of the $\mu_{s}$ values can be classified: bone and skin, the high-value group; liver and brain, the intermediate-value group; and testis and spleen, the low-value group. As we know, bone and skin protect and support the body, preventing invasion of harmful substances. These organs share similar scattering coefficients because of their analogous structure. Testis, the male reproductive organ, contains contorted seminiferous tubules, and the spleen, the largest lymphoid organ in the body, is a reticular endothelial cell organ. This similar internal reticular structure places testis and spleen in the same class. The structures of the liver and brain are neither as dense as bone and skin, nor as loose as spleen and testis; thus, their scattering coefficients are between the scattering coefficients of those two groups.
This exploratory work in quantitative SDOCT indicates that each tissue and each organ have a unique basic value of $\mu_{s}$, which appears to be an identity value of its own. With this technology, the tissue can be recognized easily without professional knowledge of tissue-image segmentation. With the endoscopic technique, quantitative SDOCT can be used as a rapid and accurate tool for issue diagnoses.

\section{Summary}

In this study, we used the scattering coefficient to quantify tissues from mouse organs (bone, skin, liver, brain, testis, and spleen). The results show that these tissues can be roughly divided into three groups. The bone and skin group has mean scattering coefficients that are 1.947 and $2.134 \mathrm{~mm}^{-1}$, 


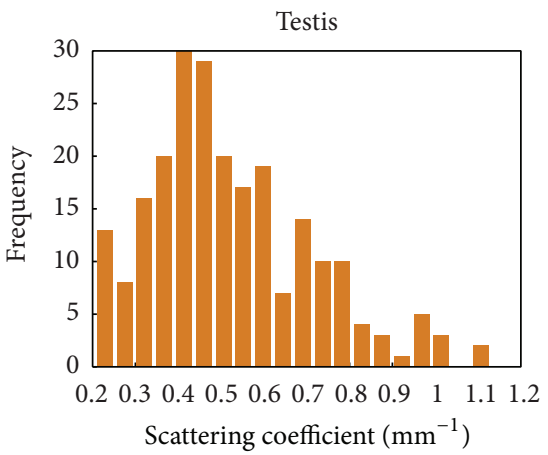

(a)

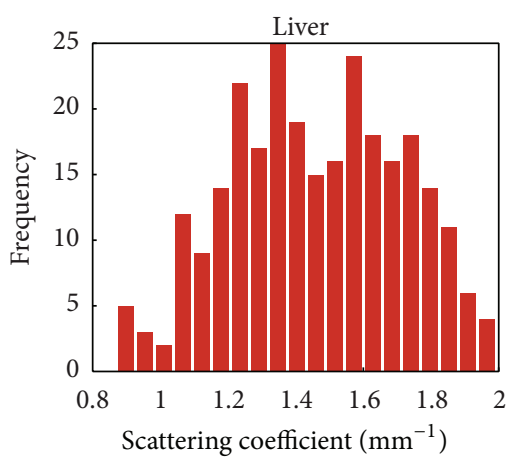

(d)

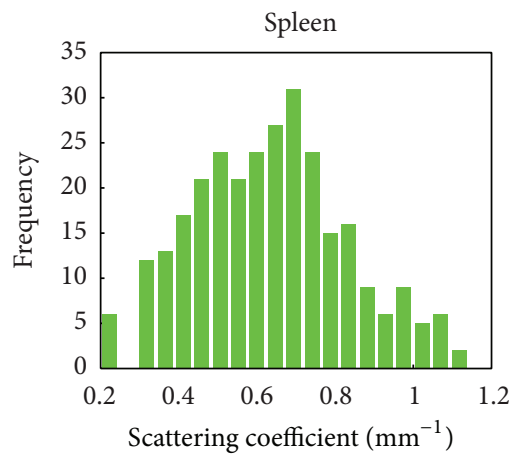

(b)

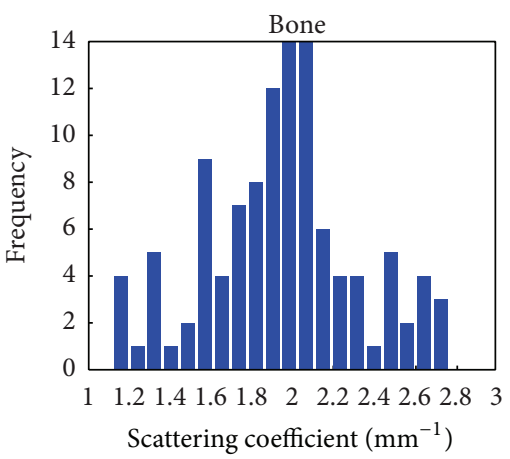

(e)

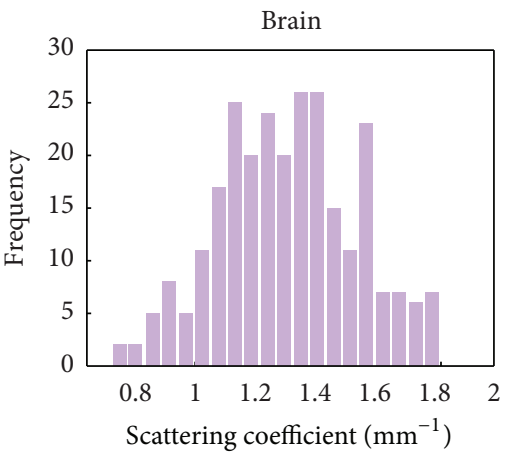

(c)

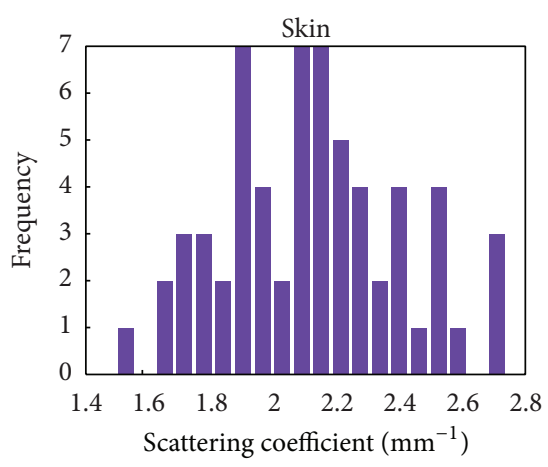

(f)

FIGURE 3: The histograms of each organ.

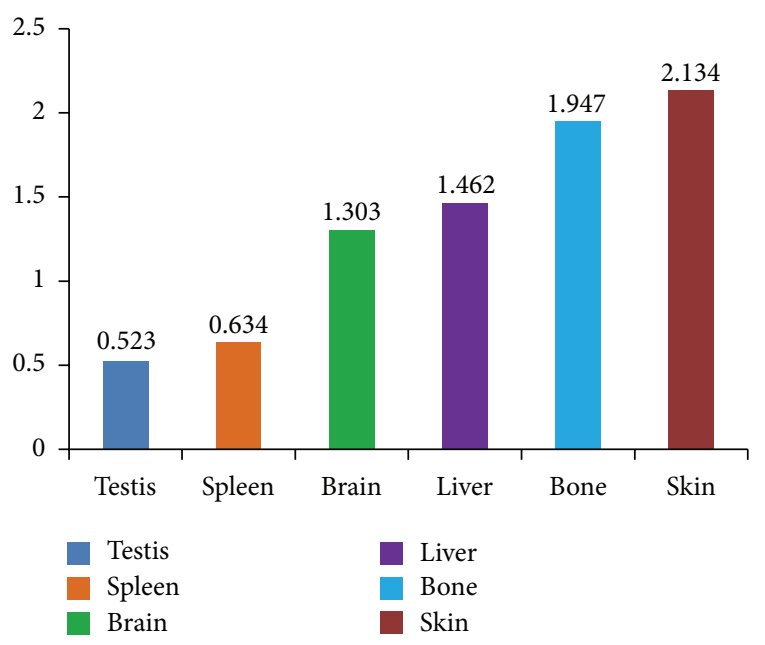

FIGURE 4: The distribution trend of organ scattering coefficients.

respectively; the liver and brain group has mean scattering coefficients of 1.303 and $1.461 \mathrm{~mm}^{-1}$; the testis and spleen group has mean scattering coefficients between 0.523 and $0.634 \mathrm{~mm}^{-1}$. SDOCT is a quantitative technology that is useful for basic tissue research and clinical diagnosis.

\section{Conflict of Interests}

The authors declare that there is no conflict of interests regarding the publication of this paper.

\section{Authors' Contribution}

Q. Q. Zhang and X. J. Wu contributed equally to this paper.

\section{Acknowledgments}

This work was partially supported by the National Nature Science Foundation of China under Grant nos. 61036013, 61138003, and 61377052. X.-C. Yuan acknowledges the support given by the Tianjin Municipal Science and Technology Commission under Grant no. 11JCZDJC 15200.

\section{References}

[1] P.-L. Hsiung, D. R. Phatak, Y. Chen, A. D. Aguirre, J. G. Fujimoto, and J. L. Connolly, "Benign and malignant lesions in the human breast depicted with ultrahigh resolution and threedimensional optical coherence tomography," Radiology, vol. 244, no. 3, pp. 865-874, 2007.

[2] D. Huang, E. A. Swanson, C. P. Lin et al., "Optical coherence tomography," Science, vol. 254, no. 5035, pp. 1178-1181, 1991.

[3] L. P. Hariri, G. T. Bonnema, K. Schmidt et al., "Laparoscopic optical coherence tomography imaging of human ovarian cancer," Gynecologic Oncology, vol. 114, no. 2, pp. 188-194, 2009.

[4] M. Kraft, H. Glanz, S. Von Gerlach, H. Wisweh, H. Lubatschowski, and C. Arens, "Clinical value of optical coherence tomography in laryngology," Head and Neck, vol. 30, no. 12, pp. 1628-1635, 2008.

[5] R. A. McLaughlin, L. Scolaro, P. Robbins, S. Hamza, C. Saunders, and D. D. Sampson, "Imaging of human lymph nodes 
using optical coherence tomography: potential for staging cancer," Cancer Research, vol. 70, no. 7, pp. 2579-2584, 2010.

[6] D. J. Faber, F. J. Van Der Meer, M. C. G. Aalders, and T. G. Van Leeuwen, "Quantitative measurement of attenuation coefficients of weakly scattering media using optical coherence tomography," Optics Express, vol. 12, no. 19, pp. 4353-4365, 2004.

[7] Z. Liu, Z. Guo, Z. Zhuang, J. Zhai, H. Xiong, and C. Zeng, "Quantitative optical coherence tomography of skin lesions induced by different ultraviolet B sources," Physics in Medicine and Biology, vol. 55, no. 20, pp. 6175-6185, 2010.

[8] S. S. Gao, A. Xia, T. Yuan et al., "Quantitative imaging of cochlear soft tissues in wild-type and hearing-impaired transgenic mice by spectral domain optical coherence tomography," Optics Express, vol. 19, no. 16, pp. 15415-15428, 2011.

[9] O. K. Adegun, P. H. Tomlins, E. Hagi-Pavli et al., "Quantitative analysis of optical coherence tomography and histopathology images of normal and dysplastic oral mucosal tissues," Lasers in Medical Science, vol. 27, no. 4, pp. 795-804, 2011.

[10] Y. Yang, T. Wang, N. C. Biswal et al., "Optical scattering coefficient estimated by optical coherence tomography correlates with collagen content in ovarian tissue," Journal of Biomedical Optics, vol. 16, no. 9, Article ID 090504, 2011.

[11] P. Lee, W. Gao, and X. Zhang, "Performance of single-scattering model versus multiple-scattering model in the determination of optical properties of biological tissue with optical coherence tomography," Applied Optics, vol. 49, no. 18, pp. 3538-3544, 2010.

[12] D. J. Faber, F. J. Van Der Meer, M. C. G. Aalders, and T. G. Van Leeuwen, "Quantitative measurement of attenuation coefficients of weakly scattering media using optical coherence tomography," Optics Express, vol. 12, no. 19, pp. 4353-4365, 2004.

[13] D. Levitz, L. Thrane, M. H. Frosz et al., "Determination of optical scattering properties of highly-scattering media in optical coherence tomography images," Optics Express, vol. 12, no. 2, pp. 249-259, 2004.

[14] D. Huang, E. A. Swanson, C. P. Lin et al., "Optical coherence tomography," Science, vol. 254, no. 5035, pp. 1178-1181, 1991.

[15] N. Nassif, B. Cense, B. H. Park et al., "In vivo human retinal imaging by ultrahigh-speed spectral domain optical coherence tomography," Optics Letters, vol. 29, no. 5, pp. 480-482, 2004.

[16] A. Unterhuber, B. Považay, B. Hermann et al., "Compact, lowcost Ti: $\mathrm{Al}_{2} \mathrm{O}_{3}$ laser for in vivo ultrahigh-resolution optical coherence tomography," Optics Letters, vol. 28, no. 11, pp. 905-907, 2003.

[17] Q. Q. Zhang, J. G. Wang, M. W. Wang et al., "A modified fractal zone plate with extended depth of focus in spectral domain optical coherence tomography," Journal of Optics, vol. 13, no. 5, Article ID 055301, 2011.

[18] C. Y. K. James, H. C. Tzu, K. N. Beng, S. G. Razul, M. Olivo, and C. J. R. Sheppard, "Concentration dependence of gold nanoshells on the enhancement of optical coherence tomography images: a quantitative study," Applied Optics, vol. 48, no. 10, pp. D96-D108, 2009.

[19] F. J. Van Der Meer, D. J. Faber, D. M. B. Sassoon, M. C. Aalders, G. Pasterkamp, and T. G. Van Leeuwen, "Localized measurement of optical attenuation coefficients of atherosclerotic plaque constituents by quantitative optical coherence tomography," IEEE Transactions on Medical Imaging, vol. 24, no. 10, pp. 13691376, 2005.

[20] J. M. Schmitt, A. Knuttel, and R. F. Bonner, "Measurement of optical properties of biological tissues by low-coherence reflectometry," Applied Optics, vol. 32, no. 30, pp. 6032-6042, 1993.
[21] J. M. Schmitt and A. Knüttel, "Model of optical coherence tomography of heterogeneous tissue," Journal of the Optical Society of America A, vol. 14, no. 6, pp. 1231-1242, 1997.

[22] H. J. van Staveren, C. J. M. Moes, J. van Marle, S. A. Prahl, and M. J. C. van Gemert, "Light scattering in Intralipid-10\% in the wavelength range of 400-1100 nm," Applied Optics, vol. 30, no. 31, pp. 4507-4514, 1991.

[23] Q. Q. Zhang, X. J. Wu, T. Tang et al., "Quantitative analysis of rectal cancer by spectral domain optical coherence tomography," Physics in Medicine and Biology, vol. 57, no. 16, pp. 52355244, 2012. 


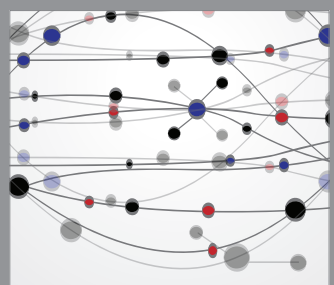

The Scientific World Journal
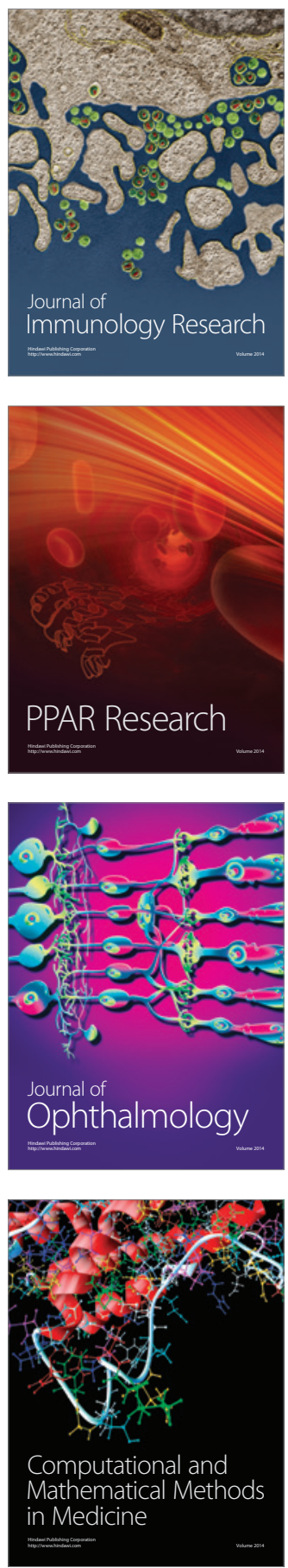

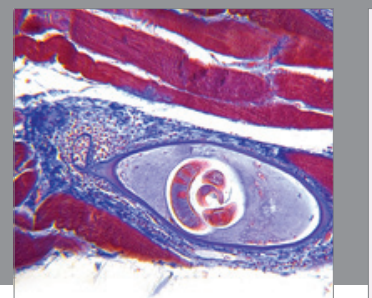

Gastroenterology

Research and Practice
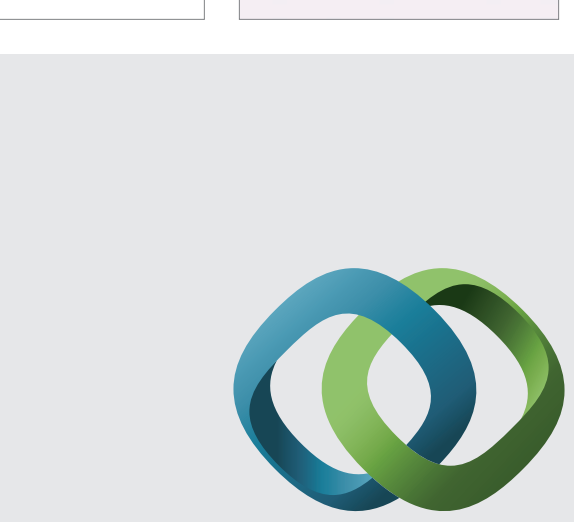

\section{Hindawi}

Submit your manuscripts at

http://www.hindawi.com
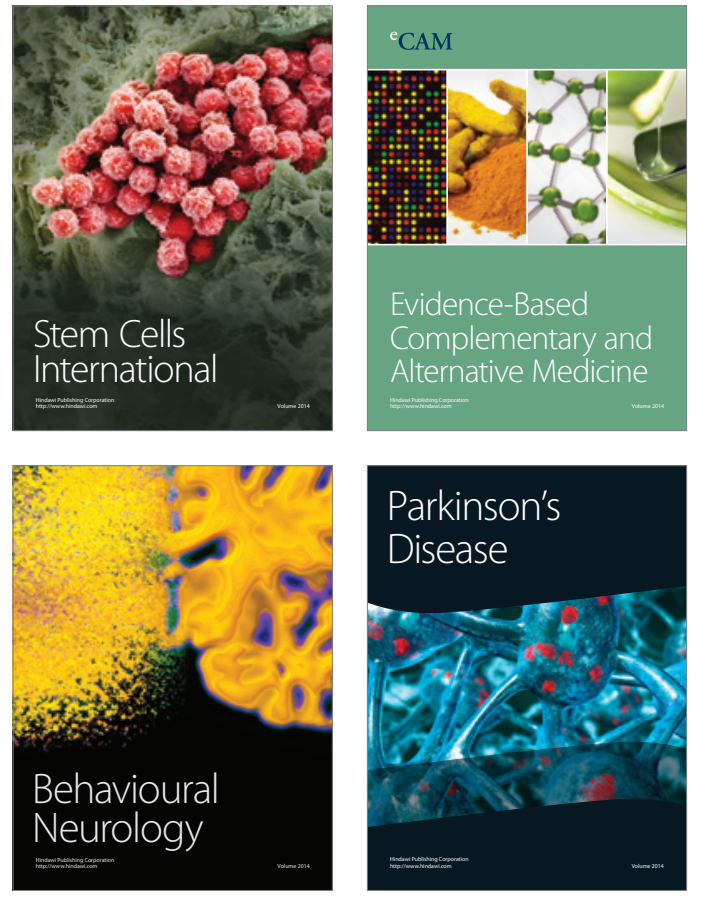
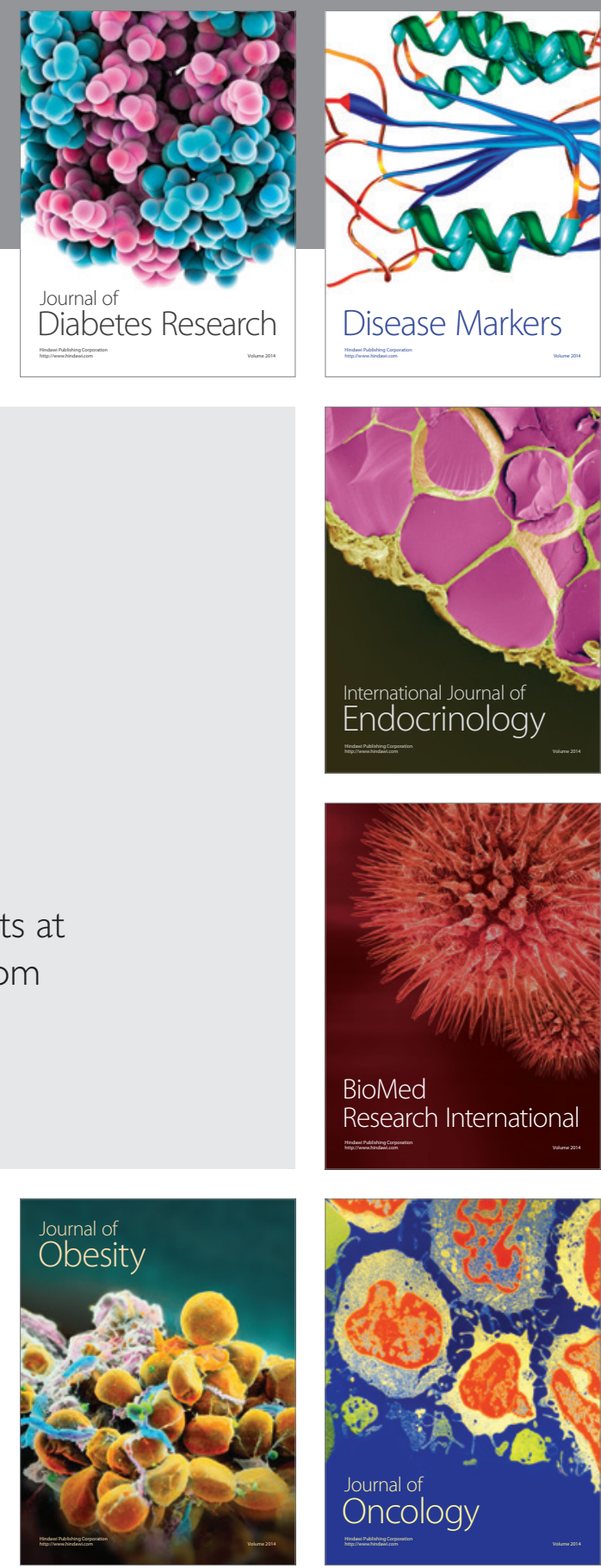

Disease Markers
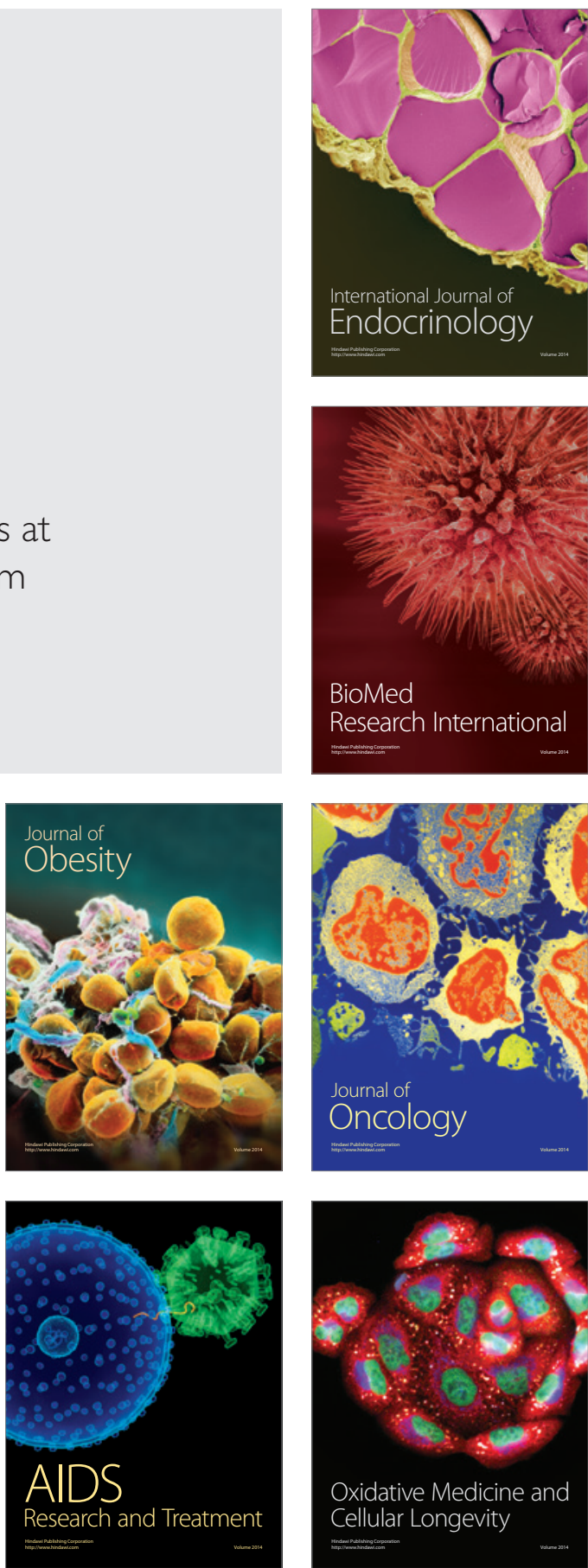